\section{Imminent Weapons in the Field of Tuberculosis Diagnostics and Management}

\author{
Anamika Gupta and Vijay Nema* \\ Department of Molecular Biology, National AIDS Research Institute, \\ Bhosari, Pune, India
}

\begin{abstract}
Tuberculosis (TB) has always been a challenge for clinicians and researchers. A lot have been done for its management and lot more is awaited. Diagnosing TB became faster with the advent and validation of Xpert ${ }^{\circledR}$ MTB/RIF (GeneXpert MTB/RIF or Xpert) and improvements over these instruments are coming to help to resolve bottlenecks. Similarly, better drugs with shorter treatment durations are long awaited and there has been some progress on this front too. This short review discusses recent advances from these fields.

Keywords: Bedaquiline; GeneXpert MTB/RIF; PaMZ

Key Message: After a long struggle with the management of tuberculosis epidemic, we are now gearing up for its end. To achieve that, more work is now focused on better diagnostics and more effective drugs.
\end{abstract}

\section{Introduction}

Worldwide, 9.6 million people are estimated to have fallen ill with tuberculosis (TB) in 2014 among which 2.3 million were from India [1]. A notable development in TB control has been achieved globally over the last two decades. The TB death rate has dropped by an estimated $47 \%$ between 1990 and 2015 but in India, there is no significant decrease in the number of incident TB cases and in case of developing Multidrug-Resistant (MDR)-TB cases, instead of any fall, the numbers are on the rise [1]. Globally a total of $1,11,000$ people started MDR-TB treatment in 2014, an increase of $14 \%$ compared with 2013 but the global data shows an average cure rate of only $50 \%$ for treated MDRTB patients. An estimated $9.7 \%$ of people with MDR-TB also have extensively drug-resistant TB (XDR-TB) until 2015, 105 countries have been reported XDR-TB cases [1].

Despite sincere efforts with well-planned strategies, TB incidence is still not falling as quickly as expected. A new efficient vaccine can help

*Corresponding author: Vijay Nema, Division of Molecular Biology, National AIDS Research Institute, 73 G MIDC Bhosari, Pune - 411026, India, Tel: +91 2027331200; E-mail: dr.vijaynema@gmail.com; vnema@icmr.org.in

Citation: Gupta A, Nema V (2017) Imminent Weapons in the Field of Tuber culosis Diagnostics and Management. $\mathrm{J}$ intern Med Prim Healthcare 2: 005.

Received: May 14, 2016; Accepted: January 19, 2017; Published: February 10, 2017 for future TB elimination programs but unfortunately, we still didn't find a new effective vaccine against TB [2]. The reasons remains, the lack of validated animal models, the way our natural immune system responds to TB infection, the antigen identification and selection and a human challenge model, as well as by the size and cost of Proof-ofConcept clinical trials.

MDR-TB and extensively drug-resistant (XDR)-TB with high mortality are the real challenges for TB control programs all over the world. About $9 \%$ of MDR-TB patients develop XDR-TB, which is even more difficult to treat [3]. The situation became worse with the emergence of Totally Drug-Resistant TB (TDR-TB) or extremely drug resistant TB (XXDR-TB). Recently, World Health Organization (WHO) established a determined post-2015 global strategy, "the End TB Strategy" [4]. This strategy outlines a 2025 milestone of $50 \%$ reduction in incidence and 75\% reduction in mortality, and an overall 2035 target of $90 \%$ reduction in incidence and $95 \%$ reduction in mortality. To achieve these goals, countries have to intensify their TB control efforts by using new TB control strategies [3].

\section{Scenario in TB diagnostics}

In developing countries like India, the availability of inexpensive drugs without need of any prescription facilitates drug-resistant TB. Further, inaccurate testing adds more onto it. The United Nations-based, Stop TB Partnership reports that India's public health sector relies mostly on smear microscopy to diagnose TB and drug-resistance testing (DST) is offered only to a small percentage of all TB patients [5].

Quick diagnosis and cure are crucial as when TB patients are not diagnosed and cured quickly, they may unknowingly spread their infection to their families and communities and exacerbating the epidemic [6]. The century-old smear microscopy and culture methods are exasperatingly insensitive (sensitivity between 50-52\%) and time-consuming, respectively $[7,8]$. Therefore, WHO had issued certain policy statements for improving diagnosis of $\mathrm{TB}$, including the use of commercial and noncommercial DST methods and implementation of molecular methods such as the Line Probe Assay (LPA) and GeneXpert MTB/RIF? LPA (Genotype MTBDRplus) is a WHO-endorsed test for the rapid detection of MTB-complex and Rifampin (RIF), Isoniazid (INH) resistance in smear-positive sputum specimens with excellent sensitivity and specificity but the use of LPA is currently limited to culture isolates and direct testing of smear-positive sputum specimens [9].

WHO endorsement of the Xpert MTB/RIF (GeneXpert MTB/RIF or Xpert) (Cepheid Inc, Sunnyvale, CA, USA), is one of the biggest advancement in TB diagnosis with a Limit of Detection (LOD) of 133 Colony Forming Unit/ml sputum only. The Xpert is a near patient, on demand novel integrated diagnostic device for the diagnosis of TB, as well as MDR-TB with high accuracy [10]. Despite transforming TB detection worldwide by making TB detection simple and quick, Xpert is constrained to identify the patients with MDR by using RIF-resistance as a surrogate. The assay cannot tell whether a patient with RIF-resistance remains INH-susceptible, and thus could still be 
treated with an INH-containing regime. The sensitivity of Xpert is only $60-86 \%$ in smear-negative TB $[11,12]$. Further, some false RIF-resistance cases have also been noticed recently, this might be due to abnormal real-time PCR curves or miss-identification of RIF-susceptible synonymous rpoB mutants as RIF-resistance. To overcome all these obstacles, the inventors of Xpert, now developed a new test the Xpert MTB/RIF Ultra (Ultra) assay, a next-generation test for TB with increased sensitivity to aid in detection of patients with smear-negative $\mathrm{TB}$, which is often associated with HIV co-infection. Ultra requires approximately 7 to 10 times less LOD as compared to Xpert. This data was presented in a reputed conference i.e., the annual Conference on Retroviruses and Opportunistic Infections (CROI), 2015 in Seattle, Washington, USA and concluded that the new Ultra assay is much more sensitive than Xpert, and is likely to be as sensitive as liquid TB culture. In addition, Ultra has better specificity than Xpert which is due to improvements in assay design. The Ultra assay should significantly increase TB detection in smear-negative patients and in patient with HIV co-infection and provide more reliable RIF-resistance detection [13]. Xpert Ultra is expected to be available to the high burden developing countries market expected sometimes later in 2016.

The available Xpert assay further cannot identify which RIF-resistant patients can be treated with a Fluoroquinolone (FQ) and/or aminoglycoside as opposed to those who have XDR-TB. Thus, patients are at risk for being either over treated or undertreated, with potentially serious consequences. Furthermore, patients with undiagnosed XDR can remain infectious for prolonged periods, further spreading their disease. Alland and co-workers from Rutgers New Jersey Medical School, USA in collaboration with Cepheid (Sunnyvale, CA, USA) are also developing an additional test to specifically call out XDR-TB i.e., Xtend-XDR assay. This assay identifies resistance to INH, the fluoroquinolones, amikacin and kanamycin, important types of resistance not detected by the Xpert assay [14]. So here, we can hope that with Ultra and Xtend-XDR assay we will be able to give clinicians a full panel of information about DR, MDR, XDR and even smear negative MDR-TB cases which will help them to prescribe appropriate regime to the patients.

Recently, WHO recommended Genotype MTBDRsl (also known as LPA for XDR-TB diagnosis) for the detection of resistance in TB to the FQs (ofloxacin, moxifloxacin and levofloxacin) and the second-line injectable drugs (SLIDs; amikacin, kanamycin and capreomycin), to treat patients with MDR-TB [15].

\section{New Treatment Options}

WHO updated its treatment guidelines for DR-TB in May 2016 and included a recommendation on the use of the shorter MDR-TB regimen under specific conditions. The new treatment regimen can be completed in 9 to 12 months, rather than the typical 18 to 24 months. In patients with RIF-resistant or MDR-TB who have not been previously treated with second-line drugs and in whom resistance to FQs and second-line injectable agents has been excluded are considered for the shorter MDR-TB regimen. The shorter regimen includes an intensive treatment phase of 4 to 6 months and consisting of four second-line drugs and a continuation phase of 5 months and composed of two drugs. The seven recommended drugs are kanamycin, moxifloxacin, prothionamide, clofazimine, pyrazinamide, high-dose INH and EMB [16].

Treatment for MDR-TB consists of second-line drugs. Many second-line drugs are toxic and have severe side effects and can further deteriorate the situation. After over 40 years, new as well as repurposed TB drugs have been introduced which include Bedaquiline (BDQ), Delamanid (DLM) and linezolid, meropenem, clavulanate, respectively along with new generation of fluoroquinolones like gatifloxacin and moxifloxacin. BDQ and DLM have been approved by the US FDA (Food and Drug Administration) and the European Medicines Agency for treatment of MDR-TB. However, the approval is conditioned due to unavailability of sufficient data on the long-term efficacy and safety of these drugs. The WHO recommends considering the addition of BDQ or DLM when an MDR-TB regimen (Rifampicin, Isoniazid, Streptomycin, Ethambutol including a fluoroquinolone and an injectable agent in addition to PZA) is not working. DLM may also be added for patients at higher risk of poor outcomes [17-19]. Bonnet and his colleagues conducted a study to identify patients who could benefit from the addition of the BDQ and DLM and found that up to two thirds of MDR-TB patients are likely to benefit from the new drugs, especially DLM [20].

Clinical trials for combinatorial therapy along with currently used drugs are in progress. In addition, trials are underway to evaluate the efficacy of a new TB treatment called PaMZ (which contains pretomanid (previously called PA-824), moxifloxacin and pyrazinamide). According to TB Alliance if these trials succeed, then TB patients might get new, shorter treatments with reduced cost. It also promises to be compatible with commonly used HIV drugs, helping the millions of people co-infected with TB/HIV [21].

\section{Conclusion}

It is extremely difficult to eradicate TB with the current portfolio of TB diagnostic tools, available medicines and vaccines. TB incidence is declining too slowly for us to reach WHO's "End TB Strategy" by 2035. We need much better tests, drugs and vaccines to really drive down the incidence of TB rapidly. Fortunately, some progress has already been made in the form of Xpert MTB/RIF for TB/DR-TB diagnosis. Xpert Ultra and Xtend XDR assays will be the imminent weapons in the field of rapid TB diagnostics and the drug like PaMZ in treatment. In addition, its cost effectiveness will make it accessible in TB endemic countries for significant reduction of the disease. Despite the developments achieved so far, much needs still to be done to improve our approach to clinical trials of new anti-TB drugs and for combinatorial therapy and regimens. We need to work together globally to contribute in whatever capacities we may and see that we throw out this problem for ever.

\section{References}

1. WHO (2015) Global tuberculosis report 2015. World Health Organization, Geneva, Switzerland.

2. Lienhardt C, Lönnroth $\mathrm{K}$, Menzies $\mathrm{D}$, Balasegaram M, Chakaya J, et al. (2016) Translational Research for Tuberculosis Elimination: Priorities, Challenges, and Actions. PLoS Med 13: 1001965

3. WHO (2016) The End Tb Strategy - Global strategy and targets for tuberculosis prevention, care and control after 2015. World Health Organization, Geneva, Switzerland.

4. WHO (2016) Treatment of drug-resistant TB: Resources - WHO treatment guidelines for drug-resistant tuberculosis 2016 update. World Health Organization, Geneva, Switzerland.

5. Sibbald B (2016) Drug-resistant tuberculosis plagues India. CMAJ 188: 322324.

6. Gupta A, Anupurba S (2015) Detection of drug resistance in Mycobacterium tuberculosis: Methods, principles and applications. Indian J Tuberc 62: 13-22. 
7. Cattamanchi A, Dowdy DW, Davis JL, Worodria W, Yoo S, et al. (2009) Sensitivity of direct versus concentrated sputum smear microscopy in HIV-infected patients suspected of having pulmonary tuberculosis. BMC Infect Dis 9: 53.

8. Chakravorty S, Boehme C, Lee J (2012) Tuberculosis Diagnostics in the New Millennium: Role in TB Identification and Control. Tuberc Res Treat 2012: 768603.

9. Nema V (2012) Tuberculosis diagnostics: Challenges and opportunities. Lung India 29: 259-266.

10. Lawn SD, Mwaba P, Bates M, Piatek A, Alexander H, et al. (2013) Advances in tuberculosis diagnostics: the Xpert MTB/RIF assay and future prospects for a point of care test. Lancet Infect Dis 13: 349-361.

11. Vadwai $V$, Boehme C, Nabeta P, Shetty A, Alland D, et al. (2011) Xpert MTB RIF: a new pillar in diagnosis of extrapulmonary tuberculosis? J Clin Microbiol 49: $2540-2545$

12. Ioannidis P, Papaventsis D, Karabela S, Nikolaou S, Panagi M, et al. (2011) Cepheid GeneXpert MTB/RIF assay for Mycobacterium tuberculosis detection and rifampin resistance identification in patients with substantial clinical indications of tuberculosis and smear-negative microscopy results. J Clin Microbiol 49: 3068-3070.

13. Alland D, Rowneki M, Smith L, Ryan J, Chancellor M, et al. (2015) Xpert MTB/ RIF Ultra: a new near-patient TB test with sensitivity equal to culture. Paper presented at: $22^{\text {nd }} \mathrm{CROI}$, Seattle, WA, USA. Abstract 91: 23-26.
14. Alland D (2015) Pre-clinical Development of an Advanced Genexpert Test for Drug Resistant Mtb. NIH, USA.

15. World Health Organization (2016) Tuberculosis Diagnostics: Molecular LineProbe Assay for the Detection of Resistance to Second-Line Anti-TB Drugs (SL-LPA). WHO, Geneva, Switzerland.

16. World Health Organization (2016) The Shorter MDR-TB Regimen. WHO, Geneva, Switzerland.

17. World Health Organization (2014) Companion handbook to the WHO guidelines for the programmatic management of drug resistant tuberculosis. WHO, Geneva, Switzerland.

18. World Health Organization (2014) The use of delamanid in the treatment of multidrug-resistant tuberculosis - Interim policy guidance. WHO, Geneva, Switzerland.

19. World Health Organization (2013) WHO Interim guidance on the use of bedaquiline to treat MDR-TB. WHO, Geneva, Switzerland.

20. Bonnet M, Bastard M, du Cros P, Khamraev A, Kimenye K, et al. (2016) Identification of patients who could benefit from bedaquiline or delamanid: a multisite MDR-TB cohort study. Int J Tuberc Lung Dis 20: 177-186.

21. Dawson R, Diacon AH, Everitt D, van Niekerk C, Donald PR, et al. (2015) Efficiency and safety of the combination of moxifloxacin, pretomanid (PA-824), and pyrazinamide during the first 8 weeks of antituberculosis treatment: a phase $2 \mathrm{~b}$, open-label, partly randomised trial in patients with drug-susceptible or drug-resistant pulmonary tuberculosis. Lancet 385: 1738-1747. 